\title{
Normalized impedance function and the straightforward inversion scheme for magnetotelluric data
}

\author{
Sri Niwas ${ }^{1}$, P K Gupta ${ }^{1}$ and V K Gaur ${ }^{2}$ \\ ${ }^{1}$ Department of Earth Sciences, Indian Institute of Technology Roorkee, Roorkee, UA 247 667, India. \\ ${ }^{2}$ Indian Institute of Astrophysics, Bangalore 560 034, India.
}

This paper investigates the performance of normalized response function obtained by normalizing the Cagniard impedance function by a suitable factor and then rotating the phase by $45^{\circ}$ to make it purely real for homogeneous half-space and equal to the square root of the half-space resistivity. Two apparent resistivity functions based on respectively the real and imaginary parts of this response function are proposed. The apparent resistivity function using the real part contains almost the same information as that yielded by the Cagniard expression while the one using the imaginary part qualitatively works as an indicator of the number of interfaces in the earth model. The linear straightforward inversion scheme (SIS), developed by the authors employing the concept of equal penetration layers, has been used to validate the proposed apparent resistivity functions. For this purpose, several synthetic and field models have been examined. Five synthetic models are studied to establish the veracity of the new functions and two well-studied published field data sets are inverted through SIS for comparison. We noticed that the new function and SIS compliment each other and lead to better understanding of the data information and model resolution.

\section{Introduction}

One objective of the magnetotelluric (MT) method is to estimate the electrical structure of the deep sedimentary basins, lower crust and upper mantle. Cagniard (1953) developed a formulation to study the response of a simple class of earth models, a stack of homogeneous and isotropic horizontal layers underlain by a uniform half-space, to a plain, quasi-static electromagnetic (EM) wave normally incident upon it. He showed that all pertinent information about the subsurface resistivity distribution is contained in the complex spectra, $E(\omega)$ and $H(\omega)$, of mutually perpendicular horizontal components of the EM field. Since then it has been a common practice to define an apparent resistivity function of the earth model in terms of the amplitude of the observed Cagniard impedance, $Z(\omega)$. Weidelt (1972) used the reciprocal response function, $C(\omega)$, designated by Parker (1980) as admittance, to define the apparent resistivity function.
Arguing that the apparent resistivity is merely a normalizing quantity with little physical significance, Spies and Eggers (1986) proposed four alternative definitions of apparent resistivity derived from the surface Cagniard impedance. They concluded, based on the criteria of (1) minimum oscillations preceding the transition and (2) maximum rate of convergence to the underlying resistivities, that the one defined with the real part of impedance exhibits the best behaviour and the one defined with the imaginary part of the impedance exhibits the worst.

Quantitative estimation of parameters of a layered earth model from observed MT field measurements is an inverse problem. The process of inverting the MT data sets, usually of inadequate quality and quantity, is beset by the endemic problems of non-uniqueness and resolution common to all ill-posed problems (Backus and Gilbert 1970; Berdichevsky and Zhadanov 1984). Bailey (1970), Weidelt (1972) and Loewenthal (1975)

Keywords. Straightforward inversion; magnetotelluric; apparent resistivity. 
have investigated the uniqueness of the problem. The inversion scheme of Weidelt (1972) is a two-stage process comprising six steps involving Laplace transformation and integral equation solution. Whittal and Oldenburg (1986) implemented this scheme for four different norms using either the Backus-Gilbert or the linear programming method for their step three. Parker (1980) extended the work of Weidelt (1972) and elegantly discussed the problem of existence and construction of inverse solutions for an incomplete and possibly imprecise data set. Parker and Whaler (1981) gave details of the algorithms that implemented the theory of Parker (1980) using a two-stage process. These non-iterative schemes are rigorous and their implementation demands knowledge of advanced mathematical analysis and numerical methods.

Gupta et al $(1996,1997)$ derived the complete set of recurrence relations for forward and inverse computations required to implement a straightforward inversion scheme (SIS) for analyzing geoelectrical data by first reducing the non-linear inverse problem to a linear one using the discretization criterion of Kunetz (1972). The linear inverse problem is then solved through the minimum norm regularized estimator in a non-iterative manner. This solution yields estimates of the coefficients of the surface impedance power series to be used in a recurrence relation, to obtain the subsurface resistivity distribution. This scheme is linear, noniterative and uses discrete inverse theory to obtain a near-continuous and smooth conductivity model. It is very simple to implement, works well even in the presence of large errors $(\sim 20 \%)$ in data and is very stable. Also it provides an approximate solution even when the data are not consistent with a truly one-dimensional model. In the present work, the SIS has been used to evaluate the MT apparent resistivity definitions described in the literature.

For better implementation of SIS the normalized response function, $S(\omega)$, Basokur (1994) and the corresponding apparent resistivity definitions have been studied. We are motivated by the Cagniard impedance representation of Fischer et al (1981) so that its phase varies symmetrically within the range $-\pi / 4$ to $+\pi / 4$. Arguing that for a given period $T$ only the structure above a certain depth matters, they proposed an inversion scheme that starts with the shortest periods of the available data set and tries to explain the observed response, specifically the apparent resistivity and phase, in terms of a two-layer structure. Shifting successively to longer periods, discrete new layers are introduced at progressively greater depth. There are many interesting features of the scheme that need a fresh look.
It has been shown that $S(\omega)$ works optimally in SIS in comparison to other response functions for illuminating the subsurface conductivity structure from inadequate and inaccurate data sets. The apparent resistivity obtained from its real component contains almost the same information as contained in the apparent resistivity of Cagniard (1953), while that obtained from the imaginary component of $S(\omega)$ is a qualitatively superior indicator of the number of layers present in the earth model.

\section{MT apparent resistivity}

For a uniform earth, the observed surface impedance is related to the true resistivity $\rho$ as

$$
Z(\omega)=\frac{E_{x}(\omega)}{H_{y}(\omega)}=\sqrt{i \omega \mu_{0} \rho} .
$$

It has a constant phase of $\pi / 4$ radian, which is independent of resistivity. This fact was used by Cagniard (1953) to define the conventional apparent resistivity $\rho_{a|Z|}$ as follows

$$
\rho_{a|Z|}=\frac{1}{\omega \mu_{0}}|Z|^{2} .
$$

Here $\omega$ is the angular frequency of EM energization and $\mu_{0}=4 \pi \cdot 10^{-7}$ Henry $/ \mathrm{m}$, is the magnetic permeability of free-space. Equation (2) ignores the phase of the surface impedance $Z(\omega)$, which for a layered earth ranges from 0 to $\pi / 2$. Spies and Eggers (1986) defined the following new pairs of alternative apparent resistivities, based on the real and imaginary parts of $Z(\omega)$,

$$
\begin{aligned}
& \rho_{a, \operatorname{Re} Z}=\frac{2}{\omega \mu_{0}}(\operatorname{Re} Z)^{2}, \\
& \rho_{a, \operatorname{Im} Z}=\frac{2}{\omega \mu_{0}}(\operatorname{Im} Z)^{2} .
\end{aligned}
$$

Weidelt (1972) worked with a different response function $C(\omega)$, referred by Parker (1980) as admittance, having unit of length and given by

$$
C(\omega)=\frac{E_{x}(\omega)}{d E_{x}(\omega) / d x} .
$$

The corresponding definition of apparent resistivity being

$$
\rho_{a|C|}=\omega \mu_{0}|C(\omega)|^{2}=\rho_{a|Z|} .
$$

We are encouraged to consider the normalized response function (Basokur 1994) defined as

$$
S(\omega)=\frac{1}{\sqrt{i \omega \mu_{0}}} \frac{E_{x}(\omega)}{H_{y}(\omega)} .
$$


Its phase varies in a desirable symmetrical range from $-\pi / 4$ to $+\pi / 4$ with zero for a uniform medium. Since $S(\omega)=\sqrt{ } \rho$ for a uniform halfspace, accordingly, we define the following pair of apparent resistivities for non-uniform earth using the real and imaginary components of $S(\omega)$ as

$$
\begin{aligned}
& \rho_{a, \operatorname{Re} S}=(\operatorname{Re} S)^{2}, \\
& \rho_{a, \operatorname{Im} S}=(\operatorname{Im} S)^{2} .
\end{aligned}
$$

It can be shown that

$$
\rho_{a, \operatorname{Re} S}+\rho_{a, \operatorname{Im} S}=\rho_{a|Z|} \cdot
$$

$S(\omega)$ can be derived from $Z(\omega)$ through a renormalization and phase rotation. Both functions contain the same amount of information. However, each gives separate stress to different aspects of information extraction due to the difference in their phases. The aspect that $\rho_{a, \operatorname{Im} S}$ in equation (8) vanishes for a uniform earth, can be used to interpret that whenever the frequency is such that the skin depth corresponds to the central region of a thick layer $\rho_{a, \operatorname{Im} S} \rightarrow 0$, while it attains maximum value at frequency for which the skin depth corresponds to interfaces. These sharp features help in identifying the number of interfaces of a non-homogeneous earth. Furthermore, even though $\rho_{a, \operatorname{Im} S}$ is non-zero for a non-homogeneous medium, it will be shown that the inequality $\rho_{a, \operatorname{Im} S} \ll \rho_{a, \operatorname{Re} S}$ generally holds. Therefore, $\rho_{a, \operatorname{Re} S} \cong \rho_{a|Z|}$ can be taken for a wide range of resistivity variation. It is this characteristic of $\rho_{a, \operatorname{Re} S}$ in $S(\omega)$, a real function, that warrants the inversion of MT data using SIS (Gupta et al 1997) and explains the motivation behind the search for an appropriate response function $S(\omega)$.

\section{Validation exercise}

\subsection{Forward computation}

Of the various apparent resistivity functions, we worked with five functions to numerically evaluate and study their performance in representing the geoelectric section: equations (2), (3), (4) based on $Z(\omega)$ and equations (7), (8) based on $S(\omega)$. The function used by Weidelt (1972) based on $C(\omega)$ is the same as (2). In particular, the function $\rho_{a, \operatorname{Im} S}$ is useful to determine the number of interfaces since it decreases to a minimum at the frequency corresponding to the skin-depth indicating center of a layer and increases at frequency corresponding to skin-depth indicating interfaces. Furthermore, $\rho_{a, \operatorname{Im} S}$ can also qualitatively distinguish between a thick or thin layer. To demonstrate these facts as well as to
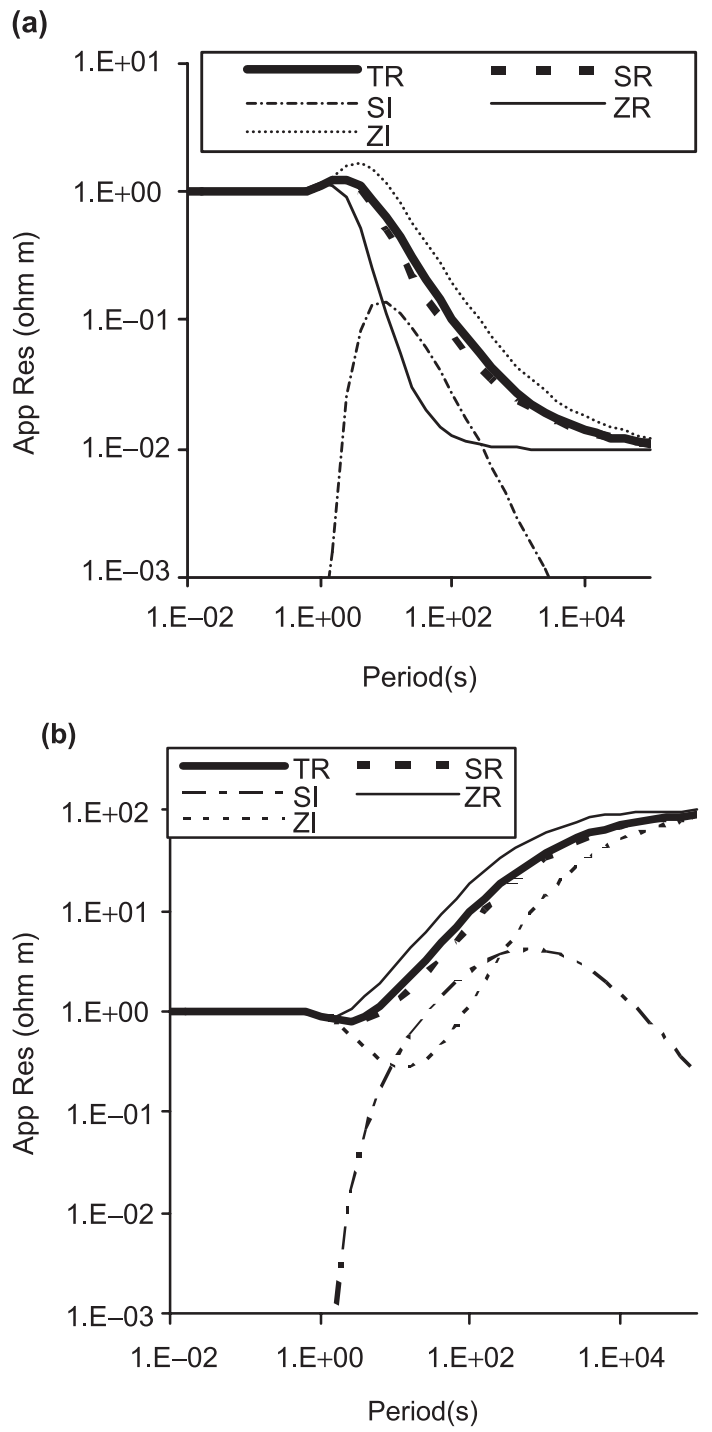

Figure 1. (a) MT apparent resistivity curves for a 2-layer earth model $\left(\rho_{1}=1 \mathrm{ohm} \mathrm{m}, \rho_{2}=100 \mathrm{ohm} \mathrm{m}, d_{1}=503 \mathrm{~m}\right)$ obtained with various definitions: $\operatorname{SR}\left(\rho_{a, \operatorname{Re} S}\right), \operatorname{SI}\left(\rho_{a, \operatorname{Im} S}\right)$, $\mathrm{ZR}\left(\rho_{a, \operatorname{Re} Z}\right), \operatorname{ZI}\left(\rho_{a, \operatorname{Im} Z}\right)$, and $\operatorname{TR}\left(\rho_{a|Z|}\right)$. (b) MT apparent resistivity curves for a 2-layer earth model $\left(\rho_{1}=1 \mathrm{ohm} \mathrm{m}\right.$, $\left.\rho_{2}=0.01 \mathrm{ohm} \mathrm{m}, \quad d_{1}=503 \mathrm{~m}\right)$ obtained with various definitions.

show that $\rho_{a, \operatorname{Im} S} \ll \rho_{a, \operatorname{Re} S}$ and $\rho_{a|Z|} \approx \rho_{a, \operatorname{Re} S}$, we made numerical computations of various apparent resistivity functions for a number of models. Figures 1(a) and 1(b) present the apparent resistivity curves $\operatorname{SR}\left(\rho_{a, \operatorname{Re} S}\right), \operatorname{SI}\left(\rho_{a, \operatorname{Im} S}\right), \operatorname{ZR}\left(\rho_{a, \operatorname{Re} Z}\right)$, $\mathrm{ZI}\left(\rho_{a, \operatorname{Im} Z}\right)$ and $\operatorname{TR}\left(\rho_{a|Z|}\right)$ for two 2-layer earth models with layer parameters: $\rho_{1}=1 \mathrm{ohm} \mathrm{m}$, $\rho_{2}=100 \mathrm{ohm} \mathrm{m}, d_{1}=503 \mathrm{~m}$ (the unit conductivity skin depth for $1 \mathrm{~s}$ period) and $\rho_{1}=1 \mathrm{ohm} \mathrm{m}$, $\rho_{2}=0.01 \mathrm{ohm} \mathrm{m}, \quad d_{1}=503 \mathrm{~m}$ respectively. Figures 2(a), and 2(b) show a graphical representation of these apparent resistivities over two, 3-layer earth models, each respectively, having a thin conducting $\left(\rho_{2}=0.01 \mathrm{ohm} \mathrm{m}, d_{2}=0.5 d_{1}\right)$ and resistive second layer $\left(\rho_{2}=100 \mathrm{ohm} \mathrm{m}\right.$, 
(a)

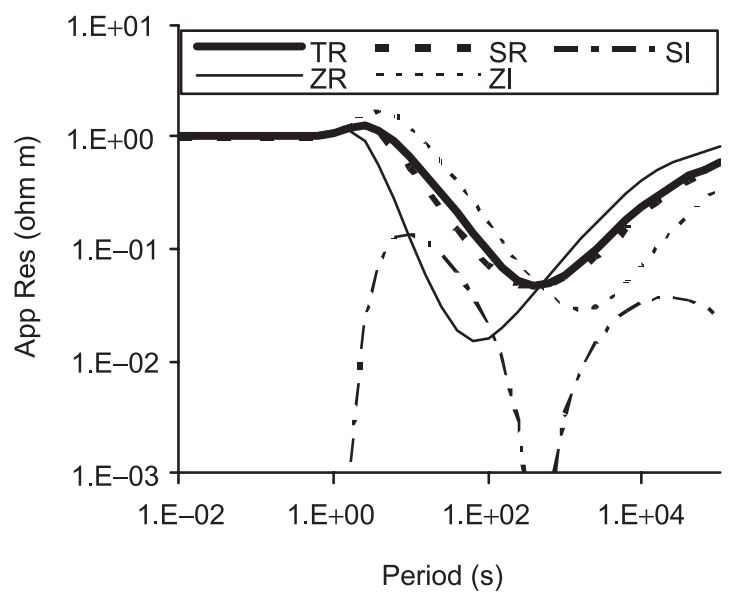

(b)

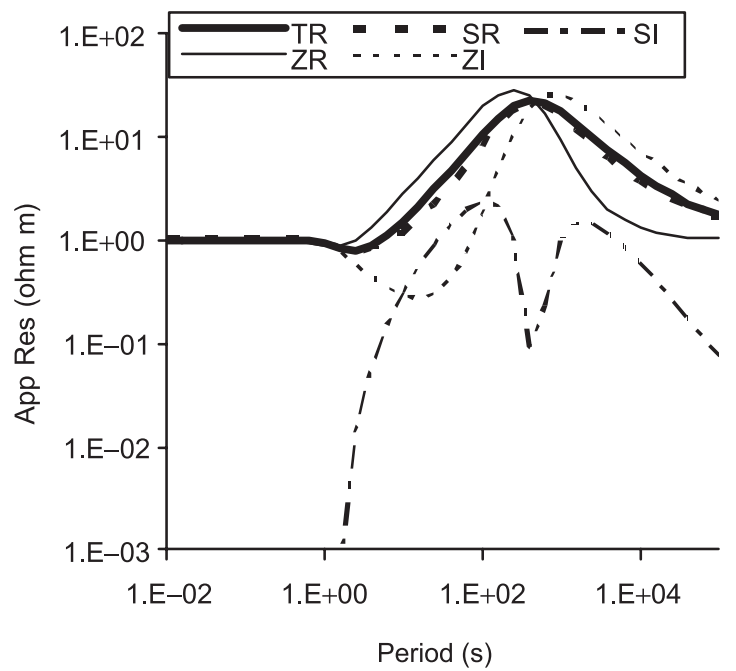

Figure 2. (a) A comparison of various MT apparent resistivity definitions for a resistive layer embedded in a uniform half-space $\left(\rho_{1}=1 \mathrm{ohm} \mathrm{m}, \rho_{2}=100 \mathrm{ohm} \mathrm{m}, \rho_{3}=1 \mathrm{ohm} \mathrm{m}\right.$, $\left.d_{1}=503 \mathrm{~m}, d_{2}=50 d_{1} \mathrm{~m}\right)$. (b) A comparison of various apparent resistivities for a conducting layer embedded in a uniform half-space $\left(\rho_{1}=10 \mathrm{ohm} \mathrm{m}, \rho_{2}=0.01 \mathrm{ohm} \mathrm{m}\right.$, $\left.\rho_{3}=1 \mathrm{ohm} \mathrm{m}, d_{1}=503 \mathrm{~m}, d_{2}=0.5 d_{1} \mathrm{~m}\right)$.

$d_{2}=50 d_{1} \mathrm{~m}$ within an otherwise uniform halfspace $\left(\rho_{1}=\rho_{3}=1 \mathrm{ohm} \mathrm{m}, d_{1}=503 \mathrm{~m}\right.$. Likewise, figure 3 shows various synthetic apparent resistivity values over a 5-layer model (Oldenburg 1990) with parameters: $\rho_{1}=250 \mathrm{ohm} \mathrm{m}, \rho_{2}=25 \mathrm{ohm} \mathrm{m}$, $\rho_{3}=100 \mathrm{ohm} \mathrm{m}, \quad \rho_{4}=10 \mathrm{ohmm}, \quad \rho_{5}=25 \mathrm{ohm} \mathrm{m}$, $d_{1}=1 \mathrm{~km}, d_{2}=2 \mathrm{~km}, d_{3}=3 \mathrm{~km}, d_{4}=4 \mathrm{~km}$. A careful examination of these synthesized data reveals, as expected from the theoretical discussion above, that each minimum of $\rho_{a, \operatorname{Im} S}$ corresponds to the center of a subsurface layer and also that $\mathrm{SR} \approx \mathrm{TR}$. Therefore, figure 1 (a) and figure $1(\mathrm{~b})$ clearly indicate a 2-layer model. Figure 2(a) and figure 2(b) likewise indicate a 3-layer case, and figure 3 is a 5 -layer case. The numerical value confirms the usefulness of SI curves in discerning the

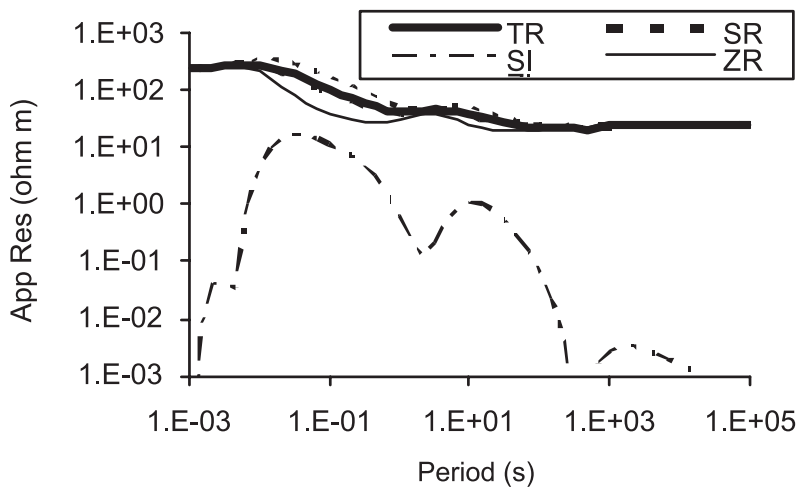

Figure 3. Various apparent resistivity curves for a 5-layer model: $\left(\rho_{1}=250 \mathrm{ohm} \mathrm{m}, \rho_{2}=25 \mathrm{ohm} \mathrm{m}, \rho_{3}=100 \mathrm{ohm} \mathrm{m}\right.$, $\rho_{4}=10 \mathrm{ohm} \mathrm{m}, \quad \rho_{5}=25 \mathrm{ohm} \mathrm{m}, \quad d_{1}=1 \mathrm{~km}, \quad d_{3}=2 \mathrm{~km}$, $\left.d_{3}=3 \mathrm{~km}, d_{4}=4 \mathrm{~km}\right)$.

number of interfaces although they do not indicate whether the layer is conducting or resistive.

All the apparent resistivity values presented here were computed using the SIS formulation at 41 data points for periods ranging from $10^{-3} \mathrm{~s}$ to $10^{5} \mathrm{~s}$ and a minimum layer thickness $(d)$ of $500 \mathrm{~m}$ which fixes, using the minimum time period value, the equal penetration layer thickness parameter $\alpha=0.0316$ (refer equation A3 of Appendix).

\subsection{Inversion}

For inversion exercises we have used the SIS algorithm (Gupta et al 1996) briefly outlined in the Appendix. In this scheme, value of the layer thickness parameter $\alpha$ is chosen judiciously keeping in mind the expected thickness and resistivity of the most conducting target layer. This is an important step in realizing the potential of SIS that is based on initial value problem. As a guideline, $\alpha$ should be $10 \%$ of the minimum layer thickness (in units of corresponding layer skin depth) that the interpreter wants to be resolved (for example a $500 \mathrm{~m}$ thick layer of resistivity $9 \mathrm{ohm} \mathrm{m}$ should have $\alpha \leq 0.15)$. Smaller values give emphasis to the shallower part while larger values give emphasis to the deeper part of the subsurface. At a given frequency, $f$, the impedance is dependent only on the conductivity of the portion above a maximum depth while the portion below this depth would influence the impedance at frequency smaller than $f$. Incidentally this seems to violate the analytic character of the complex function $Z(\omega)$ as discussed by Weidelt (1972) whereby any portion of $Z(\omega)$, if known exactly, contains information about the entire structure. However, $Z(\omega)$ is never known with complete accuracy, therefore our observation is justified.

The performance of the four apparent resistivity functions given by equations (3), (4), (7) and (8) 
Table 1. Comparative performance of the different functions using data set for the 5-layer model corrupted with different noise levels.

\begin{tabular}{cllll}
\hline Noise, (misfit), $[\mathrm{misfit}]$ & $\rho_{a, \operatorname{Re} Z}(\mathrm{ZR})$ & \multicolumn{1}{c}{$\rho_{a, \operatorname{Im} Z}(\mathrm{ZI})$} & \multicolumn{1}{c}{$\rho_{a, \operatorname{Re} S}(\mathrm{SR})$} & $\rho_{a, \operatorname{Im} S(\mathrm{SI})}$ \\
\hline$e=0 \%,\left(\varepsilon_{r}\right)\left[\varepsilon_{t}\right]$ & $(0.0)[0.114]$ & $(0.0)[0.042]$ & $(0.0)[0.085]$ & $(0.0)[0.983]$ \\
$e=0.1 \%,\left(\varepsilon_{r}\right)\left[\varepsilon_{t}\right]$ & $(0.001)[0.138]$ & $(0.002)[0.125]$ & $(0.001)[0.122]$ & $(0.032)[7.17]$ \\
$e=1.0 \%,\left(\varepsilon_{r}\right)\left[\varepsilon_{t}\right]$ & $(0.011)[0.147]$ & $(0.009)[0.087]$ & $(0.009)[0.085]$ & $(0.09)[5.88]$ \\
$e=10 \%,\left(\varepsilon_{r}\right)\left[\varepsilon_{t}\right]$ & $(0.095)[0.187]$ & $(0.090)[0.188]$ & $(0.092)[0.110]$ & $(0.299)[0.302]$ \\
$e=25 \%,\left(\varepsilon_{r}\right)\left[\varepsilon_{t}\right]$ & $(0.307)[0.317]$ & $(0.295)[0.423]$ & $(0.299)[0.302]$ & $(0.304)[0.74]$ \\
\hline
\end{tabular}

are tested by inverting the synthetic, error free data. The conventional Cagniard impedance, being complex variable, is excluded for the reason that SIS can only be applied to real functions. The SIS inverse solutions show that all of the functions yield satisfactory inverse solutions in the case of error free data. In order to study their comparative resolving power in case of noisy data, as is the case with field data, the synthesized data for each of the above function were corrupted with $0.1,1,10$ and $25 \%$ random Gaussian noise. The resulting rms errors $\varepsilon_{r}$ and $\varepsilon_{t}$ (refer to Appendix equations A14 and A15) obtained for each set during the inversion process are tabulated in table 1 . These results may be used to conclude that, of the four MT apparent resistivity functions SR and ZR are superior to the other two in the case of noisy data. SR is better in comparison to ZR and SI yields better information about layer numbers than ZI. In general, the effect of noise is rather severe on the resolution of a highly conducting deeper layer. Therefore layer 4 of the 5-layer model could not be retrieved when the data are inaccurate. Also the magnitude of peak values of layer resistivities for layers 1 and 3 are underestimated.

\section{Inversion of field data}

To further test the performance of the above response functions, we generated ZR and SR from the COPROD field data (table 2) collected at a site near Newcastleton in Britain and described by Jones and Hutton (1979). Figure 4 graphically presents these data as SR, SI and original apparent resistivity data TR. These are also consistent with the earlier observation that $\mathrm{SR} \approx \mathrm{TR}$ and that SI indicates the number of interfaces present in the earth model. Qualitatively, we should expect a 5-layer earth model as indicated by SI curve. Such a clear indication of the number of interfaces is not evident from the phase plot of $Z(\omega)$ given in figure 5. The SIS inverted model is given in figure 6 . Jones and Hutton (1979) and Constable et al (1987) have earlier inverted the COPROD data set. A comparison of
Table 2. The COPROD data set from Jones and Hutton (1979).

\begin{tabular}{ccccc}
\hline Period(s) & $\log _{10} \rho_{a}$ & $\sigma_{\log \rho}$ & $\begin{array}{c}\text { Phase } \\
(\text { degree })\end{array}$ & $\begin{array}{c}\sigma_{\text {phase }} \\
(\text { degree })\end{array}$ \\
\hline 28.5 & 2.315 & 0.0721 & 57.19 & 22.95 \\
38.5 & 2.254 & 0.0425 & 58.19 & 22.95 \\
52.0 & 2.229 & 0.0244 & 61.39 & 4.46 \\
70.5 & 2.188 & 0.0210 & 59.09 & 4.46 \\
95.5 & 2.180 & 0.0164 & 59.89 & 5.96 \\
129.0 & 2.162 & 0.0173 & 51.19 & 22.95 \\
174.6 & 2.151 & 0.0287 & 46.89 & 22.95 \\
236.2 & 2.208 & 0.0328 & 42.79 & 2.46 \\
319.6 & 2.194 & 0.0193 & 36.89 & 1.65 \\
432.5 & 2.299 & 0.0270 & 32.00 & 22.95 \\
585.1 & 2.338 & 0.0591 & 44.00 & 6.37 \\
791.7 & 2.420 & 0.0506 & 32.00 & 2.46 \\
1071.1 & 2.405 & 0.0825 & 37.59 & 22.95 \\
1449.2 & 2.308 & 0.1233 & 45.29 & 4.15 \\
1960.7 & 2.397 & 0.0927 & 50.09 & 22.95 \\
\hline
\end{tabular}

ridge-regression (Jones and Hutton 1979), JHM model; smooth (Constable et al 1987), CSM model and SIS model of COPROD data set reveals that all the significant features of the earth model are common in all the three solutions, except for the finer feature - the deep conductive region at a depth of $350 \mathrm{~km}$. The resistivity of the deep conductive zone in the JHM model is $1 \mathrm{ohm} \mathrm{m}$, in the CSM model it is higher (around $300 \mathrm{ohm} \mathrm{m}$ ) and in the SIS model, it is $27 \mathrm{ohm} \mathrm{m}$. Further, this layer is distinctly defined only in the SIS model. The layer is clearly visible independently in the SI curve of figure 4 . The misfit values of $\varepsilon_{r}$ and $\varepsilon_{t}$ obtained in SIS are 0.0322 and 0.0383 respectively while working with regression parameter $e^{2}=0.01$. It may be added here that the thumb rule to control the instability of an inverse solution is to choose the regression parameter proportional to the expected error in the data. In cases where the geological setting is at variance with the layered earth, it should be treated as geological noise and the regression parameter should be chosen as a value larger than the one likely to be chosen in case of a layered earth. The SIS solution is obtained without an 
Table 3. MT data set from Cull (1985).

\begin{tabular}{lcccc}
\hline Period(s) & $\log _{10} \rho_{a}$ & $\sigma_{\log \rho}$ & $\begin{array}{c}\text { Phase } \\
(\text { degree })\end{array}$ & $\begin{array}{c}\sigma_{\text {phase }} \\
(\text { degree })\end{array}$ \\
\hline 0.020 & 0.712 & 0.0434 & 20.50 & 4.50 \\
0.030 & 0.813 & 0.1406 & 22.00 & 19.98 \\
0.079 & 0.964 & 0.1138 & 19.28 & 16.08 \\
0.126 & 1.148 & 0.1609 & 20.14 & 11.03 \\
0.199 & 1.030 & 0.3046 & 35.65 & 23.57 \\
0.316 & 1.238 & 0.4880 & 34.44 & 20.66 \\
0.501 & 1.503 & 0.3244 & 39.71 & 16.60 \\
0.794 & 1.520 & 0.4772 & 41.90 & 23.40 \\
1.258 & 1.675 & 0.4306 & 47.28 & 16.60 \\
1.995 & 1.944 & 0.4412 & 42.78 & 16.77 \\
3.162 & 1.844 & 0.4081 & 48.50 & 18.41 \\
5.011 & 1.737 & 0.5236 & 56.87 & 18.05 \\
7.943 & 1.614 & 0.1995 & 58.80 & 17.63 \\
12.580 & 1.592 & 0.4780 & 58.88 & 18.10 \\
19.950 & 1.336 & 0.2228 & 61.44 & 18.31 \\
31.620 & 1.358 & 0.4471 & 61.90 & 16.58 \\
50.110 & 1.345 & 0.3524 & 51.48 & 25.52 \\
79.430 & 0.935 & 0.1041 & 65.50 & 10.51 \\
125.800 & 0.821 & 0.0961 & 58.20 & 23.53 \\
199.500 & 0.545 & 0.4000 & 54.00 & 20.00 \\
794.299 & 0.312 & 0.4000 & 86.00 & 30.00 \\
1258.000 & -0.107 & 0.5561 & 41.00 & 32.00 \\
1995.00 & 0.390 & 0.8612 & 58.66 & 21.63 \\
\hline & & & &
\end{tabular}

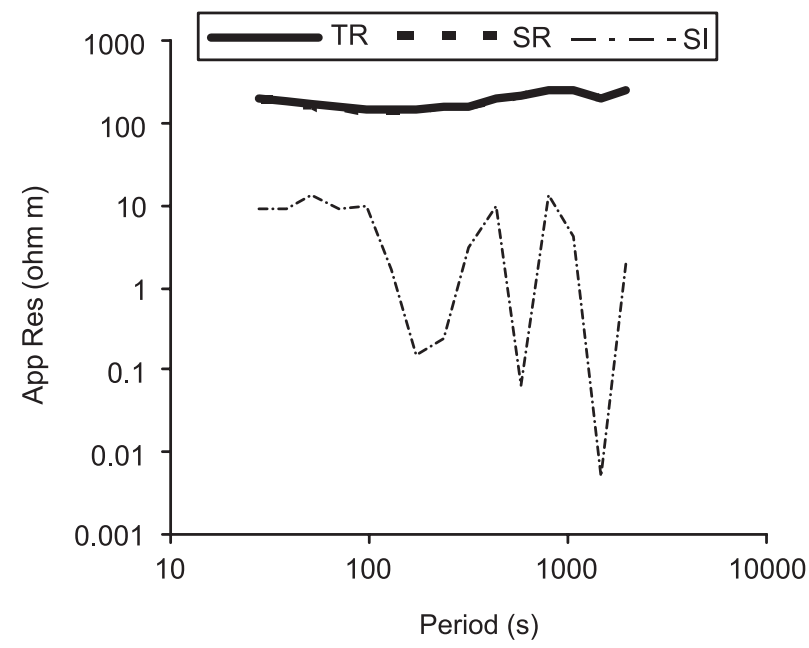

Figure 4. COPROD data, TR, of Jones and Hutton (1979) and the derived data SR and SI.

initial guess. The SIS solution using SR, thus are clearly defined in comparison with the JHM and CMS solution.

Next, the wide band MT resistivity sounding of Cull (1985) taken from a site in south central Australia is analyzed to test the versatility of the combination of SR, SI and SIS. Constable et al (1987) have given this data set. Cull (1985) inferred

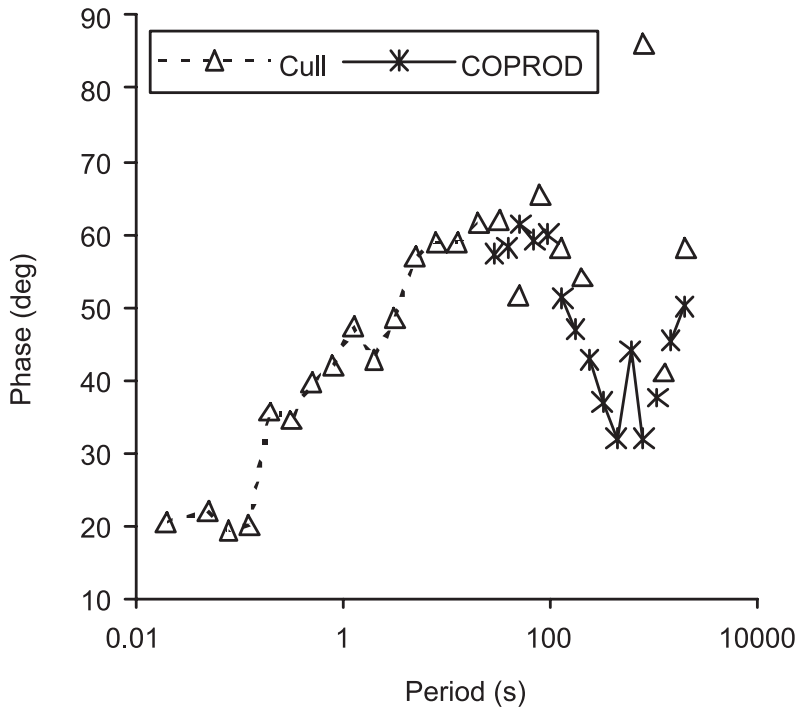

Figure 5. Phase of $Z(\omega)$ for COPROD and Cull (1985) data sets.

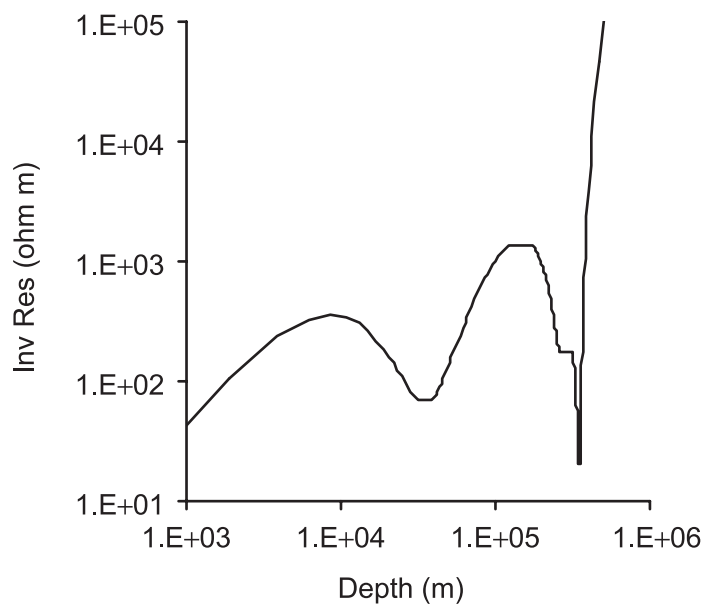

Figure 6. SIS inverted model using COPROD data, SR.

the presence of a deep conductive layer on the basis of data from several MT stations to the east of the site. Constable et al (1987) inverted this data set along with the Schlumberger sounding data set from same site individually and jointly. They found a significant difference between the joint and the individual MT models. Our choice of this data set is motivated by this observation. Figure 7 contains the data set SR, TR and SI. In this case also it confirms that $\mathrm{SR} \approx \mathrm{TR}$ and that the SI curve indicates a 4-layer configuration. Figure 5 contains the phase of $Z(\omega)$ without any indication of the number of interfaces. To clearly decipher deep and shallow structures we inverted the SR data, using SIS, with two $\alpha$ values 0.01 and 0.05 (refer figure 8). The regression parameter is fixed at $e^{2}=0.01$. It is clear that the deeper layer is not seen in the solution with $\alpha=0.01$, but shallow structure is clearly evident (figure 8). The deeper structure is distinctly 


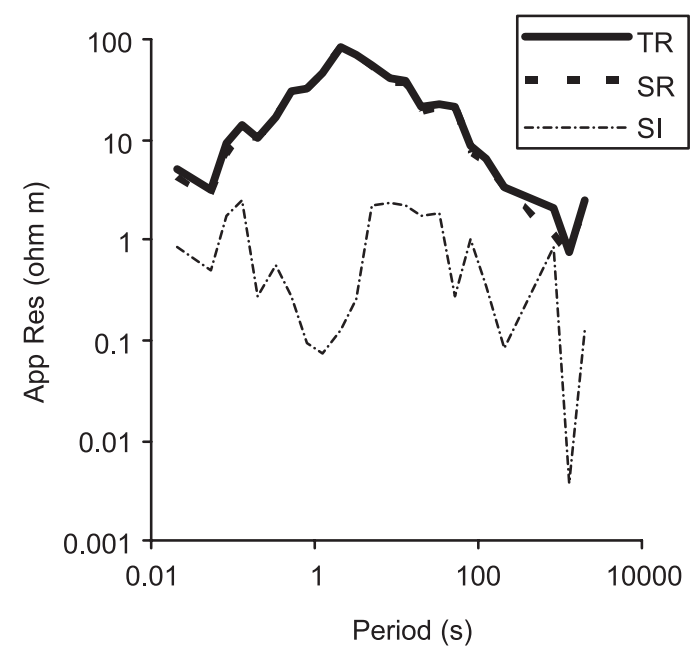

Figure 7. TR data from Cull (1985).

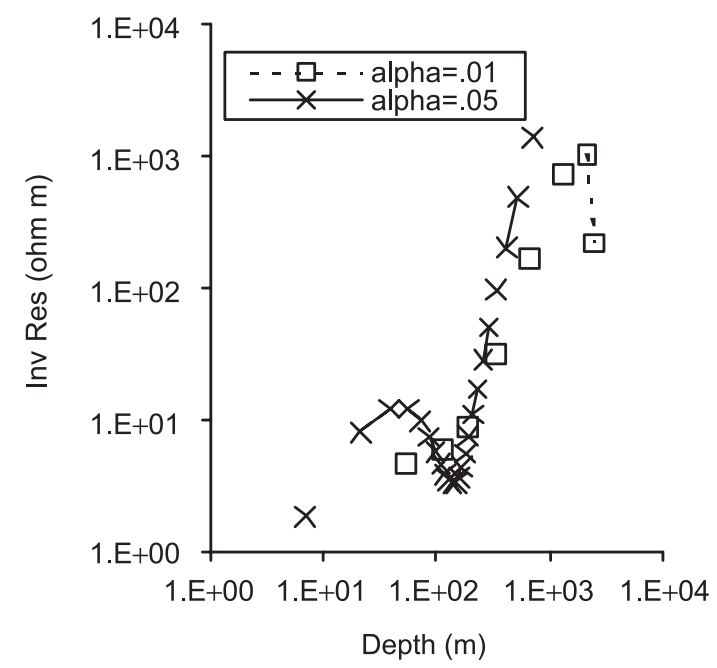

Figure 8. SIS inverted model for two $\alpha$ values using data SR from MT data of Cull (1985).

visible in the solution with $\alpha=0.05$ while the shallow structure is smoothed out. A composite model can be constructed to see both these features. This facility of resolution-guided inversion is the distinct feature of SIS. Salient features of the model obtained by Constable et al (1987) using joint inversion are recovered in the SIS model using only the MT data. The robustness of SIS is thus evident.

\section{Conclusion}

The normalized response function $S(\omega)$ and the associated two apparent resistivity functions SR and SI are studied. All the different resistivity functions contain the same amount of information, as all are derived from $E(\omega)$ and $H(\omega)$. However, due to differences in phase, different response functions give more stress to the different aspects of information and therefore, the degree of information extraction. The sharp features of the imaginary part of $S(\omega)$ help in identifying the number of interfaces. The $\operatorname{Re} S(\omega)$ is used to exploit the potential of the linear SIS formulation circumventing the hazardous requirement of an initial guess in quasi-linear inverse formulations. The SIS inversion of the SR component of two-field data sets resulted in the same number of interfaces as independently revealed by the corresponding SI curves. Judicious choice of the layer thickness unit parameter $\alpha$ controls the depth to which a conductive horizon can be detected as demonstrated for the Cull (1985) data. On the analysis of results obtained herein, we can conclude that a combination of the apparent resistivity functions SR, SI and the linear inversion scheme SIS constitute a powerful robust tool for 1D MT data inversion.

\section{Acknowledgement}

Two of the authors (SN and PKG) are thankful to the Council of Scientific and Industrial Research, Government of India for providing financial assistance in the form of a research grant.

\section{Appendix}

\section{SIS Formulation}

The surface impedance of an $n$-layered stack resting on a half-space, at the top of the $l$ th layer (Pedersen and Hermance 1986) can be written after necessary modification, as:

$$
S_{l}=\sqrt{\rho_{l}} \frac{1+R_{l} e^{-2 v_{l} d_{l}}}{1-R_{l} e^{-2 v_{l} d_{l}}}, \quad l=1,2, \ldots, n-1 .
$$

Here, $R_{l}$, the reflection function and $v_{l}$, the propagation constant are respectively given by

$$
\begin{aligned}
R_{l} & =\frac{\sqrt{\rho_{l}}-S_{l+1}}{\sqrt{\rho_{l}}+S_{l+1}}, \\
v_{l} & =\sqrt{i \omega \mu_{0} / \rho_{l}}
\end{aligned}
$$

and

$$
S_{n}=\sqrt{\rho_{n}} .
$$

The basic steps in the implementation of the SIS program (Gupta et al 1996) are:

- Parameterization of the earth model in such a way that the thickness $d_{l}$ of the $l$ th layer is expressed in units of its skin depth $\delta_{l}$ using $\alpha$ given by,

$$
\alpha=\frac{d_{l}}{\delta_{l}}=m \alpha
$$


with

$$
\delta_{l}=\sqrt{\frac{\rho_{l}}{\pi f \mu_{0}}}, \quad \alpha=\frac{d}{\delta_{\min }}
$$

and

$$
\delta_{\min }=\sqrt{\frac{\rho_{\min } t_{\min }}{\pi \mu_{0}}},
$$

$l=1,2, \ldots, n l, n l$ being the number of layers in the synthetic model.

Here the integer $m$ is the number of layers into which a given model layer is divided.

- Expansion of the reflection function at each interface derived from the following recurrence relation

$$
R_{l-1}(u)=\frac{R_{l}(u)+r_{l-1}}{1+R_{l}(u) r_{l-1}}
$$

as a power series in $u$ :

$$
R_{l}(u)=\sum_{m=1}^{\infty} R_{l m} u^{m} .
$$

Here, $R_{n-1}(u)=0, R_{n}(u)=r_{n} u ; u=e^{-2(l+i) \alpha \sqrt{f}}$, $f=\omega / 2 \pi$, and $r_{l}$ is the reflection coefficient at the interface between the $l$ th and $(l+1)$ th layer given by

$$
r_{l}=\frac{\sqrt{\rho_{l+1}}-\sqrt{\rho_{l}}}{\sqrt{\rho_{l+1}}+\sqrt{\rho_{l}}} .
$$

- Derivation of a recurrence relation by equating the coefficients of the same powers of $u$ in the power series of reflection functions of any two successive layers as

$$
\begin{aligned}
R_{l-1,1}+ & r_{l-1} ; \\
R_{l-1, m}= & \left(1-r_{l-1}^{2}\right) R_{l, m-1} \\
& -r_{l-1} \sum_{k=2}^{m-1} R_{l, m-k} R_{l-1, k} .
\end{aligned}
$$

In a similar manner, the expression for $S_{l}$ can also be written as a power series

$$
S_{l}(u)=\sum_{m=0}^{\infty} c_{l m} u^{m} .
$$

For $m>0$, the coefficients, $c_{l m}$ would be related to $R_{l m}$ as follows:

$$
\begin{aligned}
c_{l m} & =\sum_{j=0}^{m-1} R_{l, m-j} c_{l j} . \\
c_{l 0} & =\sqrt{\rho_{l}} .
\end{aligned}
$$

Equations (A3) to (A8) can be employed to compute the impedance at the air-earth interface corresponding to $l=1$. Since the coefficients $c_{l m} \mathrm{~s}$ are real, both real and imaginary components of impedance can be obtained simply by retaining in the series the real and imaginary parts of the term $u^{m}$ as

$$
\begin{aligned}
& \left(u^{m}\right)_{\operatorname{Re}}=e^{-m \beta}[\cos m \beta+\sin m \beta], \\
& \left(u^{m}\right)_{\operatorname{Im}}=e^{-m \beta}[\cos m \beta-\sin m \beta] .
\end{aligned}
$$

Here $\beta=2 \alpha \sqrt{f}$.

- Estimation of the $c_{1 m}$ 's using a regressed minimum norm estimator of the matrix form of equation (16) at air-earth interface, $U c=S_{1}$, as

$$
\hat{c}=U^{t} w ; \quad w=\left(U U^{t}+N\right)^{-1} S_{1} .
$$

Here $N$ is the data error covariance matrix. It is important to note that no truncation is employed in the evaluation of $i j$ th element of $U U^{t}$ which is obtained as

$$
\left(U U^{t}\right)_{i j}=\sum_{k=0}^{m}\left(u_{j} u_{k}\right)^{k}=\frac{1}{1-u_{i} u_{j}},
$$

and in case $E$ is not available it can be approximated by $e^{2} I, e$ being the average signal-to-noise ratio. Thus, equation (20) provides that

$$
\hat{c}_{l 0}=\sum_{l} w_{l}=\sqrt{\rho_{1}}
$$

and

$$
\hat{c}_{l m}=\sum_{l} u_{l}^{m} w_{l}, \quad m>0 .
$$

- Evaluation of layer resistivities and thickness using the inverse recurrence relations

$$
\begin{aligned}
R_{l, m-l} & =\frac{1}{\left(1-r_{l-1}^{2}\right)} \\
\times & {\left[R_{l-1, m}+r_{l-1} \sum_{k=2}^{m-1} R_{l, m-k} R_{l-1, k}\right] . }
\end{aligned}
$$

Thus the various reflection coefficients $r_{l}=R_{l 1}$ and $\rho_{l+1}=\left[\left(1+R_{l 1}\right) /\left(1-R_{l 1}\right)\right]^{2} \rho_{l}$.

Once the resistivity of the layer is obtained, thickness can readily be computed through the expression 


$$
d_{i}=\alpha \delta_{i}=\alpha \sqrt{\rho_{i} /\left(\pi f \mu_{0}\right)}, \quad i=1,2, \ldots, n l i,
$$

nli being the number of layers chosen in the inverted model.

These 5 steps solve completely the inverse MT problem in a linear fashion without any initial guess. The quality of the inverse solution is assessed by computing the relative root mean square error, $\varepsilon_{r}$ between observed $S_{1}$ and the predicted $\hat{S}_{1}=U \hat{c}$ given as

$$
\varepsilon_{r}^{2}=\sum_{i=1}^{m}\left[\frac{S_{1 i}-\hat{S}_{1 i}}{S_{1 i} \sqrt{m}}\right]^{2}
$$

and another misfit, $\varepsilon_{t}$, between the response $\tilde{S}_{1}$ computed using the inverted conductivity model and the observed $S_{1}$ given by

$$
\varepsilon_{t}^{2}=\sum_{i=1}^{m}\left[\frac{S_{1 i}-\tilde{S}_{1 i}}{S_{1 i} \sqrt{m}}\right]^{2}
$$

\section{References}

Backus G E and Gilbert J E 1970 Uniqueness in the inversion of inaccurate gross earth data; Phil. Trans. Roy. Soc. London 266 123-192.

Bailey R C 1970 Inversion of the geomagnetic induction problem; Proc. Roy. Soc. London 315 185-194.

Basokur A T 1994 Definitions of apparent resistivity for the presentation of magnetotelluric sounding data; Geophysical Prospecting 42 141-149.

Berdichevsky M N and Zhdanov M S 1984 Advanced theory of deep geomagnetic Sounding; (Elsevier, Amsterdam).

Cagniard L 1953 Basic theory of magnetotelluric method of geophysical prospecting; Geophysics 18 605-635.
Constable C C, Parker R L and Constable C G 1987 Occam's inversion: A practical algorithm for generating smooth models from electromagnetic sounding data; Geophysics 52 289-300.

Cull J P 1985 Magnetotelluric soundings over a Precambrian contact in Australia; Geophys. J. Roy. Astr. Soc. 80 661-675.

Fisher F, Schnegg P A, Pegurion M and Le Quang B V 1981 An analytic one dimensional magnetotelluric inversion scheme; Geophys. J. Roy. Astr. Soc. 67 257-278.

Gupta P K, Sri Niwas and Gaur V K 1996 Straightforward inversion scheme (SIS) for one-dimensional magnetotelluric data; Proc. Indian Acad. Sci. (Earth Planet. Sci.) $105413-429$

Gupta P K, Sri Niwas and Gaur V K 1997 Straightforward inversion of vertical electrical sounding data; Geophysics $62775-785$.

Jones A G and Hutton R 1979 A multi-station magnetotelluric study in southern Scotland I. Fieldwork, data analysis and results; Geophys. J. Astr. Soc. 56 329-349.

Kunetz G 1972 Processing and interpretation of magnetotelluric soundings; Geophysics 37 1005-1021.

Loewenthal D 1975 Theoretical uniqueness of the magnetotelluric inverse problem for equal penetration discretizable models; Geophys. J. Roy. Astr. Soc. 43 897-903.

Oldenburg D W 1990 Inversion of electromagnetic data: An overview of new techniques; Geophys. Surv. 11 231-270.

Parker R L 1980 The inverse problem of electromagnetic induction: Existence and construction of solutions based upon incomplete data; J. Geophys. Res. 85 4421-4428.

Parker R L and Whaler K A 1981 Numerical methods for establishing solutions to the inverse problem of electromagnetic induction; J. Geophys. Res. 86 9574-9584.

Pederesen J F and Hermance 1986 Least-square inversion of one-dimensional magnetotelluric data: An assessment of procedures employed by Brown University; Surv. Geophys. 8 187-231.

Spies B R and Eggers D E 1986 The use and misuse of apparent resistivity in electromagnetic method; Geophysics $\mathbf{5 1}$ $1462-1471$.

Weidelt P 1972 The inverse problem of geomagnetic induction; Z. fur. Geophys. 38 257-289.

Whittal K P and Oldenburg D W 1986 Inversion of magnetotelluric data using a practical inverse scattering formulation; Geophysics 51 383-395. 